\title{
Bistable oscillations with a self-pumped phase-conjugate mirror
}

\author{
Sze-Keung Kwong* and Amnon Yariv \\ California Institute of Technology, Pasadena, California 91125
}

Received December 12, 1985; accepted March 17, 1986

\begin{abstract}
Optical bistable oscillation beams with a self-pumped phase-conjugate mirror are reported. The results of an
\end{abstract} experimental demonstration are given, and an explanation based on the threshold of oscillation is presented.

The phenomenon of two-beam coupling in photorefractive crystals can be used to obtain self-pumped phase-conjugate reflection. ${ }^{1-3}$ One such reflector involving a ring geometry ${ }^{3}$ is shown in Fig. 1 . The oscillation takes place in the resonator consisting of the crystal and mirrors $M_{1}$ and $M_{2}$ with $P_{0}$ serving as the pump beam. We refer to this oscillation as the primary one, and it results in the appearance of a phaseconjugate reflected beam $P_{1}$. Since $P_{0}$ and $P_{1}$ are a phase-conjugate pair they should be capable of acting as pump beams for oscillation between a mirror (say $\mathrm{M}_{3}$ ) and the pumped crystal. ${ }^{4}$ We find that this is indeed the case and that oscillation in an auxiliary resonator (crystal- $\mathrm{M}_{3}$ ) takes place that is sustained by the primary oscillation in the ring resonator. We find, however, that if we set up two auxiliary resonators, the second one involving the crystal and $\mathrm{M}_{4}$, then the two secondary oscillations cannot coexist, the result being a bistable mode of oscillation.

The experimental arrangement is shown in Fig. 1. The argon-ion laser $\left(514 \mathrm{~nm}, P_{0}=10 \mathrm{~mW}\right)$ was operated in a TEM $\mathrm{TE}_{00}$ mode but in a multilongitudinal mode (without étalon) so that the coherent length $L_{c}$ of the laser beam was about $7 \mathrm{~cm}$. The length of the ring cavity for the primary oscillation was $L=34.5 \mathrm{~cm}$. The distances of the two auxiliary mirrors $\mathrm{M}_{3}$ and $\mathrm{M}_{4}$ from the crystal were $L_{1}=14 \mathrm{~cm}$ and $L_{2}=20.5 \mathrm{~cm}$, respectively. These auxiliary mirrors are used to form auxiliary oscillations with the crystal. We note that

$$
\begin{aligned}
\left|L-2 L_{i}\right|<L_{c}, & i=1,2, \\
2\left|L_{1}-L_{2}\right|>L_{c} . &
\end{aligned}
$$

These numbers are important in explaining the observed bistable behavior. Let $P_{0}$ be the power of the pumping laser beam, $P_{1}$ the power of the reflected phase-conjugate beam, $P_{2}$ the power of the beam circulating counterclockwise in the ring cavity, $P_{3}$ the power of oscillation between the crystal and mirror $\mathrm{M}_{3}$, and $P_{4}$ the power of oscillation between the crystal and mirror $\mathrm{M}_{4} \cdot \mathrm{B}_{1}-\mathrm{B}_{3}$ are beam blocks used to study the bistable oscillations. The experimental procedures and results are as follows:

1. Initially $B_{1}-B_{3}$ were inserted, i.e., in the blocking position ( and $O$ represent block and unblock in Fig. 2).
2. When $B_{1}$ was removed, the primary oscillation built up. The phase-conjugate reflectivity was about $1.26 \times 10^{-2}$. The small phase-conjugate reflectivity was due to a slight intentional misalignment of the primary oscillation produced by tilting $\mathrm{M}_{2}$ horizontally.

3. When $\mathrm{B}_{2}$ was removed, oscillation between the crystal and $\mathrm{M}_{3}$ built up, and $P_{3}$ increased. Simultaneously, $P_{1}$ increased substantially and $P_{2}$ decreased. It seems that the auxiliary oscillation at $\mathrm{M}_{3}$ dominated over the primary oscillation. The reason may be misalignment of the primary oscillation and the selfalignment of the auxiliary oscillation, so that the latter had an effectively larger coupling constant. $P_{2}$ decreased because its corresponding grating was partially washed out by the strong auxiliary oscillation beam.

4. When $\mathrm{B}_{3}$ was removed, the system remained unchanged and no oscillation between the crystal and $\mathbf{M}_{4}$ was observed.

5. When $\mathrm{B}_{2}$ was inserted, the intensity of the oscillation at $\mathrm{M}_{3}$ died out $\left(P_{3} \rightarrow 0\right)$ and $P_{1}$ decreased drastically, which shows that the major contribution to $P_{1}$ came from the oscillation between the crystal and $\mathbf{M}_{3}$. $P_{2}$ increased because of the absence of the auxiliary oscillation, and oscillation at $\mathrm{M}_{4}$ began to build up. Consequently, $P_{1}$ and $P_{4}$ increased and $P_{2}$ decreased to the steady-state value (same steady-state value as in Ref. 3).

6. When $\mathrm{B}_{2}$ was removed, the system remained unchanged and no oscillation between the crystal and $\mathrm{M}_{3}$ was observed.

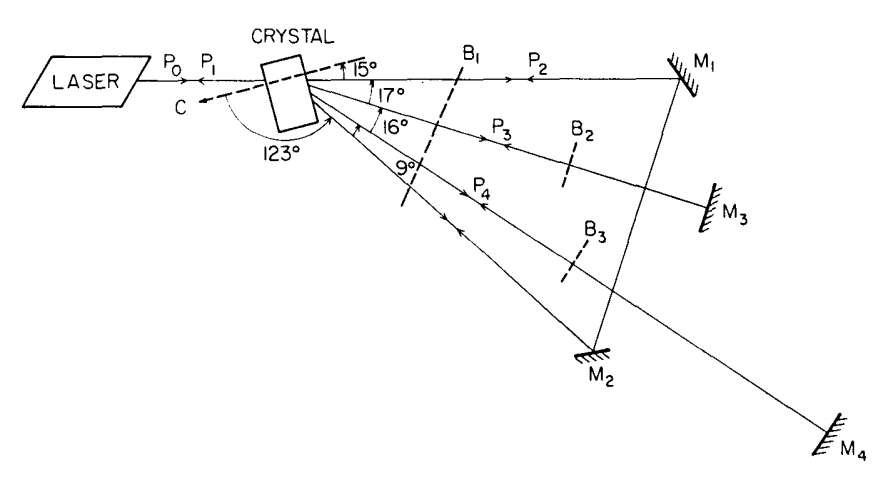

Fig. 1. Experimental arrangement to observe bistable oscillations. $M_{1}-M_{4}$ are mirrors of reflectivity $\simeq 1$. $B_{1}-B_{3}$ are beam blocks. 


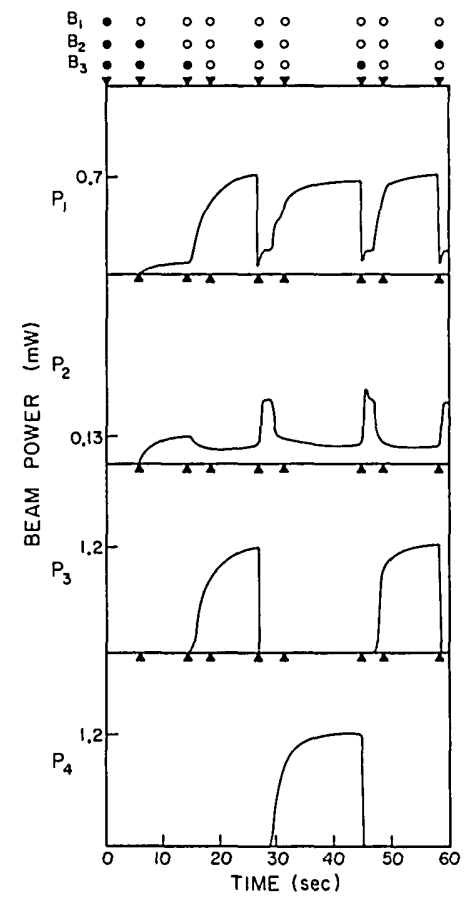

Fig. 2. $P_{1}$ is the power of the phase-conjugate beam, $P_{2}$ is the power of the beam circulating counterclockwise in the ring cavity, $P_{3}$ is the power of oscillation between the crystal and mirror $\mathrm{M}_{3}$, and $P_{4}$ is the power of oscillation between the crystal and mirror $M_{4}$. $\bullet$ and $O$ represent $B_{i}$ inserted and removed, respectively.

This cycle can be repeated indefinitely. We can consider these two auxiliary oscillations as two separate bistable states of the system: When the system, originally at one state, is disturbed, it will switch to the other state and remain in that state even when the disturbance is removed.

A complete theory of this phenomenon would require solution of eight coupled-wave equations. Nevertheless, we can still proceed to a qualitative understanding of the system by making some reasonable approximations.

A necessary condition for oscillation is that after a complete round trip the beam amplitude return to its original value, i.e., gain equals loss. Near the threshold of the auxiliary oscillation, we can assume that the oscillations are pumped by a phase-conjugate pair of beams ${ }^{5}$ in the main ring resonator with a beam ratio $r$ given approximately by $P_{2} / P_{0}$. Since the oscillation beams are weak compared with the pumping beams, at the threshold we can use the undepleted-pump approximation. The oscillation condition is then ${ }^{2}$

$$
R=\frac{1}{r}\left|\frac{J(0) e^{-\alpha l / 2}}{J(0)-1}\right|^{2} \geq \frac{1}{M T^{2}} \simeq 1.24,
$$

where

$$
J(0)=\int_{0}^{\gamma l} \frac{e^{-x} \mathrm{~d} x}{1+r^{-1} e^{-\alpha l} e^{2 \alpha x / \gamma}}
$$

and $M$ includes the loss due to the finite reflectivity of the cavity mirror and the loss due to diffraction, $T$ is the Fresnel transmittance of the crystal surface, $\gamma l$ is the coupling constant, and $\alpha l$ is the intensity absorption coefficient of the crystal. In a separate experiment, we measured $\gamma l=5.2$ and $\alpha l=1.11$ for the same beam geometry. A theoretical plot of Eq. (2) for $\gamma l=$ 5.2 and $\alpha l=1.11$ is shown in Fig. 3. With the pumping-beam ratio $r=1.26 \times 10^{-2}, R>1.24$ (point A), and oscillation can occur. Now we can explain why there is no oscillation buildup in step 4 or for step 6 . There are two reasons: (a) because of the auxiliary oscillation at $\mathrm{M}_{3}, r$ dropped to $6.63 \times 10^{-3}$, and (b) from Eq. (1), the auxiliary oscillation at $\mathrm{M}_{3}$ was incoherent with the auxiliary oscillation at $\mathrm{M}_{4}$; therefore $P_{3}$ can act as an erase beam for $P_{4}$. As a result, the effective coupling constant for the auxiliary oscillation at $\mathrm{M}_{4}$ was reduced ${ }^{6}$ :

$$
\begin{aligned}
(\gamma l)_{\text {eff }} & =\frac{(\gamma l)_{0}}{1+\frac{\text { erase beam intensity }}{\text { total beam intensity }}} \\
& \simeq \frac{(\gamma l)_{0}}{1+\frac{P_{3}}{P_{0}}}=\frac{5.2}{1+\frac{1.2}{10}}=4.64
\end{aligned}
$$

where $(\gamma l)_{0}$ is the coupling constant without erase beam. Therefore the net result is $R<1.24$ (point $\mathrm{B}$ ), and no oscillation can occur. Step 5 can also be explained in a similar way: when $B_{2}$ was inserted, the effective coupling constant increased from 4.64 to 5.2 (point $\mathrm{B}$ to point $\mathrm{C}$ ). The beam ratio $r$ increased gradually as the primary oscillation built up $\left(P_{2}\right.$ increased), and eventually at $R>1.24$ the auxiliary oscillation began to build up (point $\mathrm{C}$ to point $\mathrm{A}$ ).

If the crystal does not have any prewritten grating except that corresponding to the primary oscillation, then the system does not prefer one oscillation state over the other. A small-intensity probe beam can be used selectively to excite one of the auxiliary oscillations. This was also confirmed experimentally. This bistable oscillation has been used as a thresholding

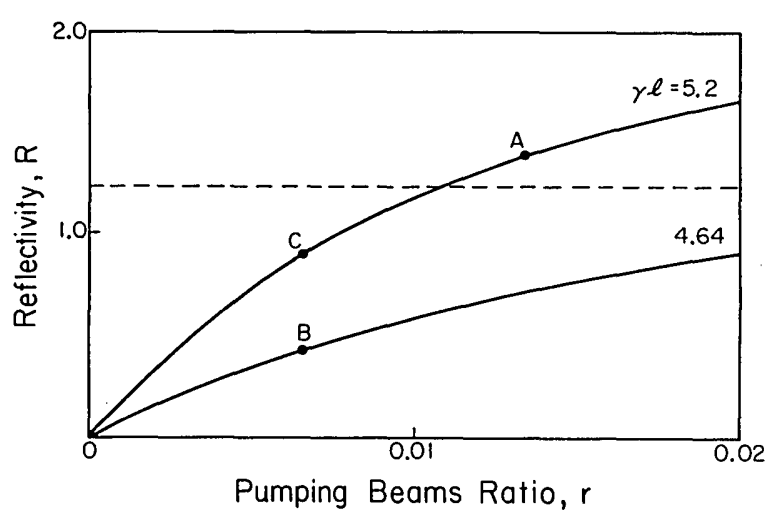

Fig. 3. Theoretical plots of reflectivity $R$ versus ring phaseconjugate mirror reflectivity $r$ for $\gamma l=4.64$ and $\gamma l=5.2$. For $R>1.24$, oscillation can occur. 
device in the first reported experimental demonstration of an all-optical associative holographic memory. ${ }^{6}$

Related work on bistability in a self-pumped phaseconjugate mirror with feedback erase beam was also reported recently. ${ }^{\text {? }}$

In summary, we have observed bistable oscillations with a self-pumped phase-conjugate mirror. An explanation based on the oscillation threshold concept has been presented. This device has potential applications in optical memory, bistable switching, and image processing.

This research was supported by the U.S. Air Force Office of Scientific Research and the U.S. Army Research Office, Durham, North Carolina.

* Present address, Ortel Corporation, 2015 West Chestnut Street, Alhambra, California 91803.

\section{References}

1. J. O. White, M. Cronin-Golomb, B. Fischer, and A. Yariv, Appl. Phys. Lett. 40, 450 (1982).

2. M. Cronin-Golomb, B. Fischer, J. O. White, and A. Yariv, IEEE J. Quantum Electron. QE-20, 12 (1984).

3. M. Cronin-Golomb, B. Fischer, J. O. White, and A. Yariv, Appl. Phys. Lett. 42, 919 (1983).

4. J. Feinberg, Opt. Lett. 8, 480 (1983).

5 . The coherent pumping condition is satisfied for the transmission grating since $\left|L-2 L_{i}\right|<L_{\mathrm{c}}, i=1,2$, from Eq. (1). A detailed discussion on the coherent conditions in ring self-pumped phase-conjugate mirrors is given by M. Cronin-Golomb, J. Paslaski, and A. Yariv, Appl. Phys. Lett. 47, 1131 (1985).

6. A. Yariv, S.-K. Kwong, and K. Kyuma, Appl. Phys. Lett. 48, 1114 (1986)

7. S.-K. Kwong, M. Cronin-Golomb, and A. Yariv, Appl. Phys. Lett. 45, 1016 (1984). 